\title{
A New QoS Provisioning Method for Adaptive Multimedia in Wireless Networks
}

\author{
F. Richard Yu, Member, IEEE, Vincent W. S. Wong, Senior Member, IEEE, and Victor C. M. Leung, Fellow, IEEE
}

\begin{abstract}
Future wireless networks are designed to support adaptive multimedia by controlling individual ongoing flows to increase or decrease their bandwidths in response to changes in traffic load. There is growing interest in quality-of-service (QoS) provisioning under this adaptive multimedia framework, in which a bandwidth adaptation algorithm needs to be used in conjunction with the call admission control algorithm. This paper presents a novel method for QoS provisioning via average reward reinforcement learning in conjunction with stochastic approximation, which can maximize the network revenue subject to several predetermined QoS constraints. Unlike other model-based algorithms (e.g., linear programming), our scheme does not require explicit state transition probabilities, and therefore, the assumptions behind the underlying system model are more realistic than those in previous schemes. In addition, when we consider the status of neighboring cells, the proposed scheme can dynamically adapt to changes in traffic condition. Moreover, the algorithm can control the bandwidth adaptation frequency effectively by accounting for the cost of bandwidth switching in the model. The effectiveness of the proposed approach is demonstrated using simulation results in adaptive multimedia wireless networks.
\end{abstract}

Index Terms-Adaptive multimedia, QoS, reinforcement learning, wireless networks.

\section{INTRODUCTION}

$\mathbf{F}$ UTURE wireless networks are expected to support adaptive multimedia applications where the bandwidth of a connection can be dynamically adjusted to adapt to the highly variable wireless communication environments. Examples of adaptive multimedia traffic include the Motion Picture Experts Group (MPEG)-4 [1] and H.263+ [2] coding for audiovisual contents. Advanced networks are designed to provide flexible radio resource allocation capabilities that efficiently support adaptive multimedia traffic. For example, third-generation (3G) universal mobile telecommunications services (UMTS) can reconfigure the bandwidth of ongoing calls [3]. However, most quality-of-service $(\mathrm{QoS})$ provisioning strategies proposed previously in the literature only consider nonadaptive traffic and nonadaptive networks [4], [5].

Manuscript received August 25, 2006; revised March 7, 2007 and June 25, 2007. The review of this paper was coordinated by Prof. B. Mark.

F. R. Yu is with Carleton School of Information Technology and the Department of Systems and Computer Engineering, Carleton University, Ottawa, ON K1S 5B6, Canada (e-mail: richard_yu@ carleton.ca).

V. W. S. Wong and V. C. M. Leung are with the Department of Electrical and Computer Engineering, The University of British Columbia, Vancouver, BC V6T 1Z4, Canada (e-mail: vincentw@ece.ubc.ca; vleung@ece.ubc.ca).

Color versions of one or more of the figures in this paper are available online at http://ieeexplore.ieee.org.

Digital Object Identifier 10.1109/TVT.2007.907023
Under the adaptive multimedia framework, a bandwidth adaptation (BA) algorithm needs to be used in conjunction with the connection admission control (CAC) algorithm for QoS provisioning. CAC decides the admission or rejection of new and handoff calls, whereas BA reallocates the bandwidth of ongoing calls. Recently, QoS provisioning for adaptive multimedia services in cellular wireless networks has been a very active area of research [6]-[16]. A channel subrating scheme for telephony services is proposed in [6]. In [7], an analytical model is derived for one class of adaptive service. The extension of these schemes that are designed for one traffic class to the case of multiple traffic classes in real cellular wireless networks may not be an easy task. Talukdar et al. [8] studied the tradeoffs between network overload and fairness in BA for multiple classes of adaptive multimedia. A near-optimal scheme was proposed in [9]. Markov decision process formulation and linear programming were used in [10]. Degradation ratio and degradation ratio degree are considered in [11]. Zaruba et al. [12] propose an excellent approach to use a simulated annealing algorithm to find the optimal call-mix selection. The Q-learning algorithm is used in [13] and [14]. The shortcomings of [6][14] are that only the status of the local cell is considered in QoS provisioning. However, due to increasing handoffs between cells that are shrinking in size, the status of neighboring cells has an increased influence on the QoS of the local cell in future multimedia cellular wireless networks [17], and therefore, information on neighboring cell traffic is very important for the effectiveness of QoS provisioning methods that can adapt to changes in the traffic pattern [5]. The status information of neighboring cells is considered in [15] and [16]; however, only one class of traffic is studied, and they do not consider maximizing network revenue.

This paper introduces a novel average reward reinforcement learning (RL) approach in conjunction with stochastic approximation to solve the QoS provisioning problem for adaptive multimedia in cellular wireless networks, which aims to maximize the network revenue while satisfying several predetermined QoS constraints. The novelties of the proposed scheme are as follows.

- The proposed scheme takes into account the effects of the status of neighboring cells on multiple classes of traffic, enabling it to dynamically adapt to changes in traffic condition.

- The underlying assumptions of the proposed solution are more realistic than those in previous proposals. Particularly, our method does not need prior knowledge of system state transition probabilities, which are very difficult to 
estimate in practice due to irregular network topology, a different propagation environment, and random user mobility.

- With the use of stochastic approximation, the proposed scheme can efficiently handle problems with large state spaces and action sets. Because there will be several classes of traffic in future mobile multimedia networks, and each class of traffic has several bandwidth levels, the state spaces and action sets are very large in formulating the QoS provisioning problems in practical networks, which makes these problems very difficult to solve using model-based algorithms (e.g., linear programming).

- The algorithm can control the adaptation frequency effectively by accounting for the cost of BA in the model, which is not done in [13] or [14]. It is observed in [7] and [8] that frequent bandwidth switching among different levels may consume a lot of resources and may be even worse than a large degradation ratio. The proposed scheme can control the adaptation frequency more effectively than previous schemes.

- Handoff dropping probability, average allocated bandwidth, and intraclass fairness are considered simultaneously as QoS constraints in our scheme and can be guaranteed.

- The proposed scheme allows action space to be effectively traded off with state space. As mentioned in [10], solutions with a large action space may hinder its deployment in real systems. With the approach of trading off action space with state space, the large action space problem in QoS provisioning can be solved.

RL has previously been used to solve CAC and routing problems in wireline networks [18], [19], the channel allocation problem in wireless networks [20], [21], the handover dropping control problem [22], and the multirate transmission control problem [23] in nonadaptive wireless networks. This paper focuses on applications of RL to solve the QoS provisioning problem in adaptive cellular wireless networks, which is different from the problems addressed in [18]-[23]. In addition, previous RL applications [13], [14], [18]-[23] are focused on the discounted sum of rewards as the optimality metric. However, with discounted RL algorithms, one has to know the value of the discount factor, which may not be available in QoS provision problems. In addition, performance measures in QoS provision problems may not suitably be described in economic terms. Hence, it may be preferable to compare policies based on time averaged expected reward rather than expected total discounted reward. Therefore, we use the average reward RL method to the QoS provisioning problem in this paper.

We compare our scheme with two existing nonadaptive and adaptive QoS provisioning schemes for adaptive multimedia in cellular wireless networks. Extensive simulation results show that the proposed scheme outperforms the others by maximizing the network revenue while satisfying the QoS constraints.

The rest of this paper is organized as follows. Section II describes QoS provisioning problems in the adaptive framework. Section III describes the average reward RL algorithm. Our new approach to solve the QoS provisioning problem using average reward RL is presented in Section IV. Section V discusses some implementation issues. Section VI presents and discusses the simulation results. Finally, we conclude this study in Section VII.

\section{QoS PROVISIONING IN ADAPTIVE FrAMEWORK}

\section{A. Adaptive Multimedia Applications}

Adaptive multimedia applications were originally introduced in wireline networks, where congestion can cause fluctuations in the availability of network resources, thereby resulting in severe degradation of QoS. Many techniques are proposed such as the adaptation of compression parameters [24] and layered coding [25] to overcome this problem. In wireless networks, because bandwidth fluctuations are much more severe, it is interesting to consider the use of adaptive multimedia.

In adaptive multimedia applications, a multimedia connection or stream can dynamically change its bandwidth requirement throughout its lifetime. For example, when the layered coding technique is used, a raw video sequence can be compressed into several layers [25], say, three layers, consisting of a base layer and two enhancement layers. The base layer can be independently decoded to provide basic video quality; the enhancement layers can only be decoded together with the base layer to further refine the quality of the base layer. Therefore, a video stream compressed into three layers can adapt to three levels of bandwidth usage.

\section{B. Adaptive Cellular Wireless Networks}

Due to the severe fluctuation of resources in wireless links, the ability of terminals or traffic sources to adapt to the communication environment is very important in future cellular wireless networks. For example, in UMTS systems, a radio bearer established for a call can be dynamically reconfigured during the call session [3]. Radio bearer information in UMTS includes most of the information in layers 2 and 1 for that call, e.g., radio link control (RLC), power control, spreading factor, diversity, and the like. When the radio bearer is reconfigured, the bandwidth of a call can be changed dynamically during a call session.

\section{QoS Provisioning Functions and Constraints}

In this paper, we consider two important functions for QoS provisioning: CAC and BA. The problem of QoS provisioning in an adaptive multimedia framework is how to define CAC and BA policies to maximize the long-term network revenue and guarantee QoS constraints. To reduce network signaling overhead, we assume that BA is exercised only when a call arrival or departure occurs. That is, BA will not be used when congestion occurs briefly due to channel fading. Lowlevel mechanisms, such as error-correction coding and efficient packet scheduling are usually used to handle brief throughput variations of wireless links.

Smaller cells (microcells/picocells) will be employed in future cellular wireless networks to increase capacity. Therefore, the number of handoffs during a call's lifetime is likely to be 
increased and the status of neighboring cells has an increased influence on the QoS of the local cell. In order to adapt to changes in traffic pattern, the status information of neighboring cells should be considered in QoS provisioning.

We consider three QoS constraints in this paper. Because forced call terminations due to handoff dropping are generally more objectionable than new call blocking, an important calllevel QoS constraint in cellular wireless networks is $P_{h d}$ : the probability of handoff dropping. As it is impractical to eliminate handoff call dropping completely, the best one could do is to keep $P_{h d}$ below a target level. In addition, although adaptive applications can tolerate decreased bandwidth, it is desirable for some applications to have a bound on the average allocated bandwidth. Therefore, we need another QoS parameter to quantify the average bandwidth received by a call. The normalized average allocated bandwidth of class $i$ calls, denoted as $A B_{i}$, is the ratio of the average bandwidth received by class $i$ calls to the bandwidth with undegraded service. In order to guarantee the QoS of adaptive multimedia $A B_{i}$ should be kept above a target value. Finally, due to BA, some calls may operate at very high bandwidth levels, whereas some calls within the same class may operate at very low bandwidth levels. This is undesirable from users' perspective. Therefore, the QoS provisioning scheme should be fair to all calls within one class, and intraclass fairness is defined as another QoS constraint in this paper. These constraints will be formulated in Section IV.

We formulate the QoS provisioning problem as a semiMarkov decision process (SMDP) [26], which is more general than a Markov decision process used in [13] and [14]. Traditional model-based solutions (e.g., linear programming) to SMDP suffer from the curse of dimensionality and the curse of modeling. Therefore, we use the average reward reinforcement learning to solve the problem.

\section{Average Reward Reinforcement Learning}

Recently, RL has become a topic of intensive research as an alternative approach to solve SMDPs. This method has two distinct advantages over model-based methods. The first is that it can handle problems with complex transitions. Second, RL can integrate within it various function approximation methods (e.g., neural networks), which can be used to approximate the value function over large state and action spaces.

Most of the published research in RL is focused on the discounted sum of rewards as the optimality metric. Qlearning [27] is one of the most popular discounted reward RL algorithms. These techniques, however, cannot extend automatically to the average reward criterion. In QoS provisioning problems, the discount factor may not be available, and performance measures may not suitably be described in economic terms. Hence, it may be preferable to compare policies based on time averaged expected reward rather than expected total discounted reward. Discounted RL methods, on average, reward problems can lead to suboptimal behavior and may converge much more slowly than average reward RL methods [28]. An algorithm for average reward RL called the semi-Markov average reward technique (SMART) [28]-[32] has emerged recently. The convergence analysis of this algorithm is given in [32], and it has been successfully applied to production inventory [29] and airline seat allocation [31] problems. We use this average reward RL method to solve the QoS provisioning problem for adaptive wireless multimedia in this paper.

\section{Solving Average Reward SMDP by RL}

In this section, we describe the average reward SMDP. The optimality equation is introduced. We then describe the reinforcement learning approach.

\section{A. Average Reward Semi-Markov Decision Process}

For a SMDP, let $S$ be a finite set of states and $A$ be a set of possible actions. In state $s \in S$, when an action $a \in A$ is chosen, a lump sum reward of $k(s, a)$ is received. Further accrual of reward occurs at a rate $c\left(s^{\prime}, s, a\right), s^{\prime} \in S$ for the time the process remains in state $s^{\prime}$ between decision epochs. The expected reward between two decision epochs, given that the system is in state $s$ and $a$ is chosen at the first decision epoch, may be expressed as

$$
r(s, a)=k(s, a)+E\left\{\int_{0}^{\tau} c\left(W_{t}, s, a\right) d t\right\}
$$

where $\tau$ is the transition time to the second decision epoch, $W_{t}$ denotes the state of the natural process, and $E$ denotes the expectation.

Starting from state $s$ at time 0 and using a policy $\pi$, the average reward $g^{\pi}(s)$ can be given as

$$
g^{\pi}(s)=\lim _{N \rightarrow \infty} \frac{E_{s}^{\pi}\left\{\sum_{n=0}^{N}\left[k\left(s_{n}, a_{n}\right)+\int_{\sigma_{n}}^{\sigma_{n+1}} c\left(W_{t}, s_{n}, a_{n}\right) d t\right]\right\}}{E_{s}^{\pi}\left[\sum_{n=0}^{N} \tau_{n}\right]}
$$

where $\sigma_{n}$ represents the time of the $(n+1)$ th decision epoch, $\tau_{n}=\sigma_{n+1}-\sigma_{n}$, and $E_{s}^{\pi}$ denotes the expectation with respect to policy $\pi$ and initial state $s$.

The Bellman optimality equation for SMDP [26] can be stated as follows.

Theorem 1: For any finite unichain SMDP, there exists a scalar $g^{*}$ and a value function $R^{*}$ satisfying the system of equations

$$
R^{*}(s)=\max _{a \in A}\left[r(s, a)-g^{*} q(s, a)+\sum_{s^{\prime} \in S} P_{s s^{\prime}}(a) R^{*}\left(s^{\prime}\right)\right]
$$

where $q(s, a)$ is the average sojourn time in state $s$ when action $a$ is taken in it, and $P_{s s^{\prime}}(a)$ is the probability of transition from state $s$ to state $s^{\prime}$ under action $a$.

For a proof of Theorem 1, see [26, ch. 11]. 


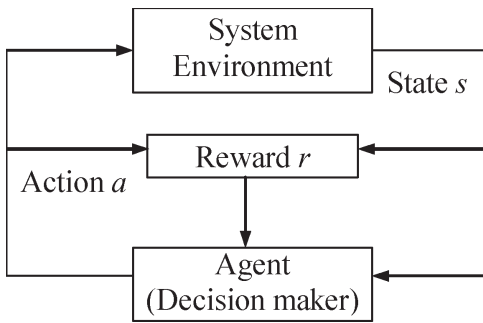

Fig. 1. Reinforcement learning model.

\section{B. Solution Using Reinforcement Learning}

In the RL model depicted in Fig. 1, a learning agent selects an action for the system that leads the system along a unique path till another decision-making state is encountered. At this time, the system needs to consult with the learning agent for the next state. During a state transition, the agent gathers information about the new state, the immediate reward, and the time spent during the state transition, based on which the agent updates its knowledge base using an algorithm and selects the next action. The process is repeated, and the learning agent continues to improve its performance.

Average reward RL uses the action value representation that is similar to its counterpart: Q-learning. The action value $R^{\pi}(s, a)$ represents the average adjusted value of choosing an action $a$ in state $s$ once and then following policy $\pi$ subsequently [28]. Let $R^{*}(s, a)$ be the average adjusted value by choosing actions optimally. The Bellman equation for average reward SMDPs (3) can be rewritten as

$$
R^{*}(s, a)=r(s, a)-g^{*} q(s, a)+\sum_{s^{\prime} \in S} P_{s s^{\prime}}(a) \max _{a^{\prime} \in A} R^{*}\left(s^{\prime}, a^{\prime}\right) .
$$

The optimal policy is $\pi^{*}(s)=\arg \max _{a \in A} R^{*}(s, a)$. The average reward RL algorithm estimates action values on-line using a temporal difference method and then uses them to define a policy.

The action value of state-action pair $(s, a)$ visited at the $n$th decision making epoch is updated as follows. Assume that action $a$ in state $s$ results in a system transition to $s^{\prime}$ at the subsequent decision epoch; then

$$
\begin{aligned}
& R_{\text {new }}(s, a)=\left(1-\alpha_{n}\right) R_{\text {old }}(s, a) \\
& \quad+\alpha_{n}\left[r_{\text {act }}\left(s^{\prime}, s, a\right)-\rho_{n} \tau_{n}+\max _{a^{\prime} \in A} R_{\text {old }}\left(s^{\prime}, a^{\prime}\right)\right]
\end{aligned}
$$

where $\alpha_{n}$ is the learning rate parameter for updating of the action value of a state-action pair of the $n$th decision epoch, and $r_{a c t}\left(s^{\prime}, s, a\right)$ is the actual cumulative reward earned between two successive decision epochs starting in state $s$ (with action $a$ ) and ending in state $s^{\prime}$. The reward rate $\rho_{n}$ is calculated as

$$
\rho_{n}=\left(1-\beta_{n-1}\right) \rho_{n-1}+\beta_{n-1} \frac{T(n-1) \rho_{n}+r_{a c t}\left(s^{\prime}, s, a\right)}{T(n)}
$$

where $T(n)$ denotes the sum of the time spent in all states visited till the $n$th epoch, and $\beta_{n-1}$ is the learning rate parameter. If each action is executed in each state an infinite number of times on an infinite run and $\alpha_{n}$ and $\beta_{n}$ are decayed appropriately, the above learning algorithm will converge to the optimality [30].

\section{FORMULATION OF QOS PROVISIONING IN ADAPTIVE FRAMEWORK}

In adaptive multimedia cellular wireless networks, we assume that call arrivals at a given cell, including new and handoff calls, follow a Poisson distribution. We further assume that each call needs a service time that is exponentially distributed and independent of the interarrival time distribution. The QoS provisioning problem for adaptive multimedia can be formulated as an SMDP. In order to utilize the average reward RL algorithm, it is necessary to identify the system state, actions, rewards, and constraints. The exploration scheme and the method to trade off action space with state space are also described in this section.

\section{A. System States}

Assume that there are $K$ classes of services in the network. A class $i$ call uses bandwidth among $\left\{b_{i 1}, b_{i 2}, \ldots, b_{i j}, \ldots, b_{i N_{i}}\right\}$, where $b_{i j}<b_{i(j+1)}$, where $i=1,2, \ldots, K, j=1,2, \ldots, N_{i}$, and $N_{i}$ is the highest bandwidth level which can be used by class $i$ call. At random times, an event $e$ can occur in a cell $c$, where $e$ is a new call arrival, a handoff call arrival, a call termination, or a call handoff to a neighboring cell. At this time, cell $c$ is in a particular configuration $x$ defined by the number of each type of ongoing calls in cell $c$. Let $\mathbf{x}=$ $\left(x_{11}, x_{12}, \ldots, x_{i j}, \ldots, x_{K N_{K}}\right)$, where $x_{i j}$ denotes the number of ongoing calls of class $i$ using bandwidth $b_{i j}$ in cell $c$ for $1 \leq i \leq K$ and $1 \leq j \leq N_{i}$. Because the status of neighboring cells is important for QoS provisioning, we also consider it in the state description. The status of neighboring cells $y$ can be defined as the number of each type of ongoing calls in all neighboring cells of cell $c$. Let $\mathbf{y}=\left(y_{11}, y_{12}, \ldots, y_{i j}, \ldots, y_{K N_{K}}\right)$, where $y_{i j}$ denotes the number of ongoing calls of class $i$ using bandwidth $b_{i j}$ in all neighboring cells of cell $c$. We assume that the status of neighboring cells is available in cell $c$ by the exchange of status information between cells. Note that this assumption is common among dynamic QoS provisioning schemes [5]. The configurations and the event together determine the state $s=(\mathbf{x}, \mathbf{y}, e)$.

We assume that each cell has a fixed channel capacity $C$ and that cell $c$ has $M$ neighboring cells. The state space is defined as

$$
\begin{array}{r}
S=\left\{s=(\mathbf{x}, \mathbf{y}, e): \sum_{i=1}^{K} \sum_{j=1}^{N_{i}} x_{i j} b_{i j} \leq C ; \sum_{i=1}^{K} \sum_{j=1}^{N_{i}} y_{i j} b_{i j} \leq M C\right. \\
\left.1 \leq i \leq K, \quad 1 \leq j \leq N_{i}\right\} .
\end{array}
$$

\section{B. Actions}

An action must be chosen according to the state when an event occurs. We use $a=\left(a_{a}, \mathbf{a}_{d}, \mathbf{a}_{u}\right)$ to denote an action, where $a_{a}$ stands for the admission decision, i.e., admit 
$\left(a_{a}=1\right)$, reject $\left(a_{a}=0\right)$, or no action due to call departures $\left(a_{a}=-1\right)$, ad stands for the action of bandwidth degradation, and au stands for the action of bandwidth upgrade. Action $\mathbf{a}_{d}$ has the form

$$
\begin{aligned}
\mathbf{a}_{d}=\left\{\left(d_{12}^{1}, \ldots, d_{i j}^{n}, \ldots, d_{K N_{k}}^{N_{k}-1}\right)\right. & \\
& \left.\quad 1 \leq i \leq K, \quad 1<j \leq N_{i}, \quad 1 \leq n<j\right\}
\end{aligned}
$$

where $d_{i j}^{n}$ denotes the number of ongoing class $i$ calls using bandwidth $b_{i j}$ that are degraded to bandwidth $b_{i n}$. Action $\mathbf{a}_{u}$ has the form

$$
\begin{aligned}
\mathbf{a}_{u}=\left\{\left(u_{11}^{2}, \ldots, u_{i j}^{n}, \ldots, u_{K N_{k}-1}^{N_{k}}\right)\right. & \\
& \left.\quad 1 \leq i \leq K, \quad 1 \leq j<N_{i}, \quad j<n \leq N_{i}\right\}
\end{aligned}
$$

where $u_{i j}^{n}$ denotes the number of ongoing class $i$ calls using bandwidth $b_{i j}$ that are upgraded to bandwidth $b_{i n}$.

\section{Rewards}

The network earns deterministic revenue due to the carried traffic in the cell. On the other hand, extra signaling overhead is required for $\mathrm{BA}$, which will consume radio and wireline bandwidth, as well as the battery power in the mobile. It is observed in [7] and [8] that frequent bandwidth switching among different levels may consume a lot of resources and may be even worse than a large degradation ratio. Thus, there is a trade-off between the network resources utilized by the calls and the signaling and processing load incurred by BA operation. We use a function to model the cost due to the action of BA. The definition of the cost function depends on specific traffic, user terminal, and network architecture in real networks. One intuitive definition is that the cost is proportional to the number of BA operations, which is used in this paper.

Let $r_{i j}$ be the reward rate of class $i$ call using bandwidth $b_{i j}, c_{a}$ be the cost of one BA operation, and $N_{a}(a)$ be the total number of BA operations in action $a$. The actual cumulative reward $r_{a c t}\left(s^{\prime}, s, a\right)$ between two successive decision epochs starting in state $s$ (with action $a$ ) and ending in state $s^{\prime}$ can be calculated as

$$
r_{a c t}\left(s^{\prime}, s, a\right)=\tau_{a c t}\left(s^{\prime}, s, a\right) \sum_{i=1}^{K} \sum_{j=1}^{N_{i}} x_{i j}^{\prime} r_{i j}-N_{a}(a) c_{a}
$$

where $\tau_{a c t}\left(s^{\prime}, s, a\right)$ is the actual sojourn time between the decision epochs.

By formulating the cost of the BA operation in the model, we can control the adaptation operation frequency effectively. Note that all ongoing calls in the cell, including those that have been degraded or upgraded, contribute to the reward $r_{a c t}\left(s^{\prime}, s, a\right)$. Therefore, we do not need an extra term to formulate the penalty related to the bandwidth degradation.

\section{Constraints}

As mentioned in Section II, the first QoS constraint is related to the handoff dropping probability. Let $P_{h d}(n)$ be the measured handoff dropping ratio upon the $n$th call arrival and $T P_{h d}$ denote the target maximum allowed handoff dropping probability. The constraint associated with $P_{h d}$ can be formulated as

$$
\lim _{N \rightarrow \infty} \frac{\sum_{n=0}^{N} P_{h d}(s) \tau_{n}}{\sum_{n=0}^{N} \tau_{n}} \leq T P_{h d}
$$

We use the Lagrange multiplier formulation to relate the constrained optimization to an unconstrained optimization [33]. To fit into this formulation, we need a new state descriptor $\bar{s}=\left(N_{h r}, N_{h d}, \tau, s\right)$, where $N_{h r}$ and $N_{h d}$ are the total number of handoff call requests and handoff call drops, respectively, $\tau$ is the time interval between the last and the current decision epochs, and $s$ is the original state descriptor. Quantified values of $P_{h d}=N_{h d} / N_{h r}$ and $\tau$ are used to make the sate space finite. A Lagrange multiplier $\lambda$ is used for the parameterized reward

$$
\bar{r}_{a c t}\left(\bar{s}^{\prime}, \bar{s}, a\right)=r_{a c t}\left(\bar{s}^{\prime}, \bar{s}, a\right)-\lambda z\left(\bar{s}^{\prime}, \bar{s}, a\right)
$$

where $r_{a c t}\left(\bar{s}^{\prime}, \bar{s}, a\right)$ is the original reward function, and $z\left(\bar{s}^{\prime}, \bar{s}, a\right)=P_{h d}(\bar{s}) \tau_{a c t}\left(\bar{s}^{\prime}, \bar{s}, a\right)$ is the cost function associated with the constraint. Lemma 3.1 of [34] shows that the original reward, the new reward, and the cost function are monotone nonincreasing in $\lambda$. This monotonicity property associated with $\lambda$ facilitates the search for a suitable $\lambda$ [34, Lemma 3.3]. The suitable $\lambda$ is chosen so that the constraint in (11) is met.

The second QoS constraint is the normalized average allocated bandwidth of class $i$ calls $A B^{i}$. $A B^{i}$ can be defined as the mean of $B^{i} / b_{i N_{i}}$ over all class $i$ calls in the current cell, where $B^{i}$ denotes the bandwidth allocated to a class $i$ call.

$$
A B^{i}=\frac{\sum_{j=1}^{N_{i}} x_{i j} b_{i j}}{b_{i N_{i}} \sum_{j=1}^{N_{i}} x_{i j}}, i=1,2, \ldots, K
$$

where $A B^{i}$ should be kept larger than the target value $T A B^{i}$ : $A B^{i} \geq T A B^{i}, i=1,2, \ldots, K$.

The third QoS constraint is the intraclass fairness constraint, which can be defined in many ways. In this paper, we use the variance of $B^{i} / b_{i N_{i}}$ over all class $i$ calls in the current cell $V B^{i}$ to characterize the intraclass fairness

$$
\begin{aligned}
& V B^{i}= \operatorname{var}\left(\frac{B^{i}}{b_{i N_{i}}}\right) \\
&=\frac{\sum_{j=1}^{N_{i}} x_{i j} \sum_{j=1}^{N_{i}} x_{i j}\left(b_{i j}\right)^{2}-\left(\sum_{j=1}^{N_{i}} x_{i j} b_{i j}\right)^{2}}{b_{i N_{i}}\left(\sum_{j=1}^{N_{i}} x_{i j}\right)^{2}} \\
& i=1,2, \ldots, K .
\end{aligned}
$$


$V B^{i}$ reflects the difference between the bandwidths of individual class $i$ calls and the average bandwidth. For absolute fairness, $V B^{i}$ should be kept to zero all the time. However, this is very difficult to achieve in practice as bandwidth is adjusted in discrete steps. Therefore, it is better to keep $V B^{i}$ below a target value $V B^{i}: V B^{i} \leq T V B^{i}, i=1,2, \ldots, K$.

$A B^{i}$ and $V B^{i}$ are intrinsic properties of a state. With the current state and action information $(\bar{s}, a)$, we can forecast $A B^{i}$ and $V B^{i}$ in the next state $\bar{s}^{\prime}, A B^{i}\left(\bar{s}^{\prime}\right)$ and $V B^{i}\left(\bar{s}^{\prime}\right)$. If $A B^{i}\left(\bar{s}^{\prime}\right) \geq T A B^{i}$ and $V B^{i}\left(\bar{s}^{\prime}\right) \leq T V B^{i}, i=1,2, \ldots, K$, the action is feasible. Otherwise, this action should be eliminated from the feasible action set $A(\bar{s})$.

For a general MDP with $M$ constraints, the optimal policy for at most $M$ of the states is randomized [35]. However, model-based algorithms (e.g., linear programming) have to be employed to derive such policy, which is impractical for the QoS provisioning problem considered in this paper. Because randomizations are needed at most $M$ states, which is much smaller than the total number of states, the loss of optimality is minimal. For the above reasons, and to avoid the complications of randomization, we concentrate on nonrandomized policies in this paper.

\section{E. Exploration}

Each action should be executed in each state an infinite number of times to guarantee the convergence of a RL algorithm. This is called exploration [36]. Exploration plays an important role in ensuring that all the states of the underlying Markov chain are visited by the system and all the potentially beneficial actions in each state are tried out. Therefore, with a small probability $p_{n}$ upon the $n$th decision-making epoch, decisions other than that with the highest action value should be taken.

In this paper, we use the Darken-Chang-Moody search-thenconverge procedure [37] to decay the learning rates $\alpha_{n}, \beta_{n}$ and the exploration rate $p_{n}$. In the following expression, $\Theta$ can be substituted by $\alpha, \beta$, and $p$ for learning and exploration, respectively. $\Theta_{n}=\Theta_{0} /\left(1+\zeta_{n}\right)$, where $\zeta_{n}=n^{2} /\left(\Theta_{r}+n\right)$, and $\Theta_{0}$ and $\Theta_{r}$ are constants.

\section{F. Trading Off Action Space Complexity With State Space Complexity}

The action space in our formulation is quite large. It may be very time consuming for the algorithm to converge. A method to trade off action space complexity with state space complexity [36, ch. 6] can be used. We can reduce the complexity of the control space by increasing the complexity of the state space. The potential advantages of doing this are that the action space will be reduced and the extra state space complexity may still be dealt with by using the function approximation described in Section V.

For an action $a=\left(a_{a}, d_{12}^{1}, \ldots, d_{i j}^{n}, \ldots, d_{K N_{k}}^{N_{k}-1}\right)$, there are, at most, $W=1+\sum_{i=1}^{K} \sum_{j=2}^{N_{i}}(j-1)$ components. At a given state $s$, the action a can be broken down into the sequence of the $W$ controls $a_{a}, d_{12}^{1}, \ldots, d_{i j}^{n}, \ldots, d_{K N_{k}}^{N_{k}-1}$ and introduce some artificial intermediate "states" $\left(\bar{s}, a_{a}\right)$, $\left(\bar{s}, a_{a}, d_{12}^{1}\right), \ldots,\left(\bar{s}, a_{a}, d_{12}^{1}, \ldots, d_{i j}^{n}, \ldots, d_{K N_{k}}^{N_{k}-1}\right)$. The choice of the last control component $d_{K N_{k}}^{N_{k}-1}$ makes the transition to the next state. This way, the action space is simplified at the expense of introducing $W-1$ additional layers of states and $W-1$ additional action values $R\left(\bar{s}, a_{a}\right)$, $R\left(\bar{s}, a_{a}, d_{12}^{1}\right), \ldots, R\left(\bar{s}, a_{a}, d_{12}^{1}, \ldots, d_{i j}^{n}, \ldots, d_{K N_{k}}^{N_{k}-2}\right)$, in addition to $R\left(\bar{s}, a_{a}, d_{12}^{1}, \ldots, d_{i j}^{n}, \ldots, d_{K N_{k}}^{N_{k}-1}\right)$. The increase in size of the state space can be dealt with by using function approximation. In addition, the above procedure can be revised to leave the order subject to choice.

\section{Algorithm Implementation}

\section{A. Neural Network Representation of Action Values}

In practice, an important issue is how to store the action value $R(s, a)$. There are several approaches, among which the lookup table is the most straightforward method to represent action values. A lookup table representation means that a separate variable $R(s, a)$ is kept in memory for each state-action pair $R(s, a)$. This method will be prohibitive when the number of state-action pairs becomes large because memory requirement can be huge. Approximate representation should be used to break the curse of dimensionality in the face of very large state spaces. Neural network is an efficient method to represent the action values. A popular neural network architecture is the multilayer perceptron (MLP) with a single hidden layer [36]. There are a number of methods available for neural network training [36]. In this paper, incremental training is applied to the network in a supervised fashion using the back-propagation algorithm available in the Matlab simulation tool. The action value is updated when we update the neural network. During training, both network inputs and target outputs are used. An input pattern is applied to the network to generate an output, which is compared to the corresponding target output to produce an error that is propagated back through the network. The network weights are adjusted to minimize the sum of the errors squared.

\section{B. Structure and Pseudo-Code}

The structure of the RL-based QoS provisioning scheme is shown in Fig. 2. A pseudo-code description of the proposed scheme is given in Fig. 3. When an event (either a call arrival or departure) occurs, a state $s$ is identified by getting the status of the local and neighboring cells. According to the state $s$, a set of feasible actions $\{a\}$ is found. The state and action information is fed into the neural network to get the action values. The action with the largest action value is chosen with probability $1-p_{n}$. Otherwise, exploration is performed, and an action is chosen randomly. When the next event occurs, the action value is updated, and the process is repeated.

\section{Simulation Results AND Discussions}

We used a cellular network of 19 cells, as shown in Fig. 4, in our simulations. Each cell has a fixed bandwidth of $2 \mathrm{Mb} / \mathrm{s}$. Wraparound is applied to the edge cells so that each cell has six neighbors. In Table I, we describe the parameters in the 


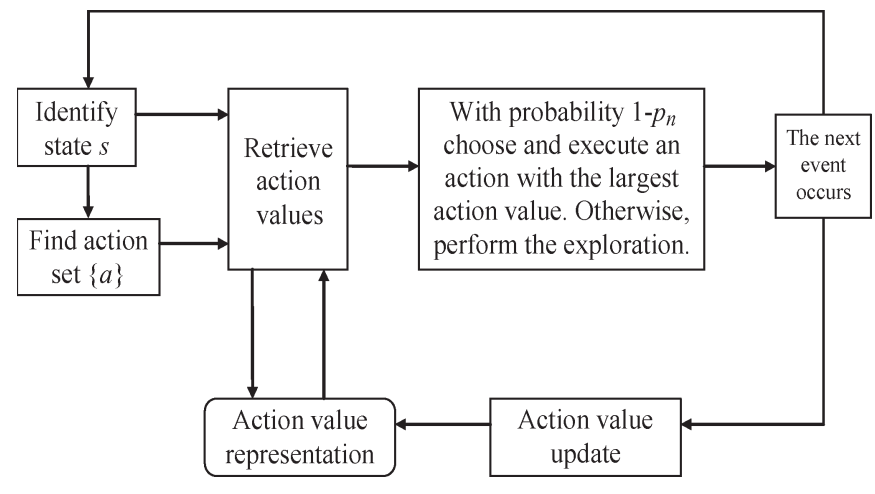

Fig. 2. Structure of the QoS provisioning scheme.

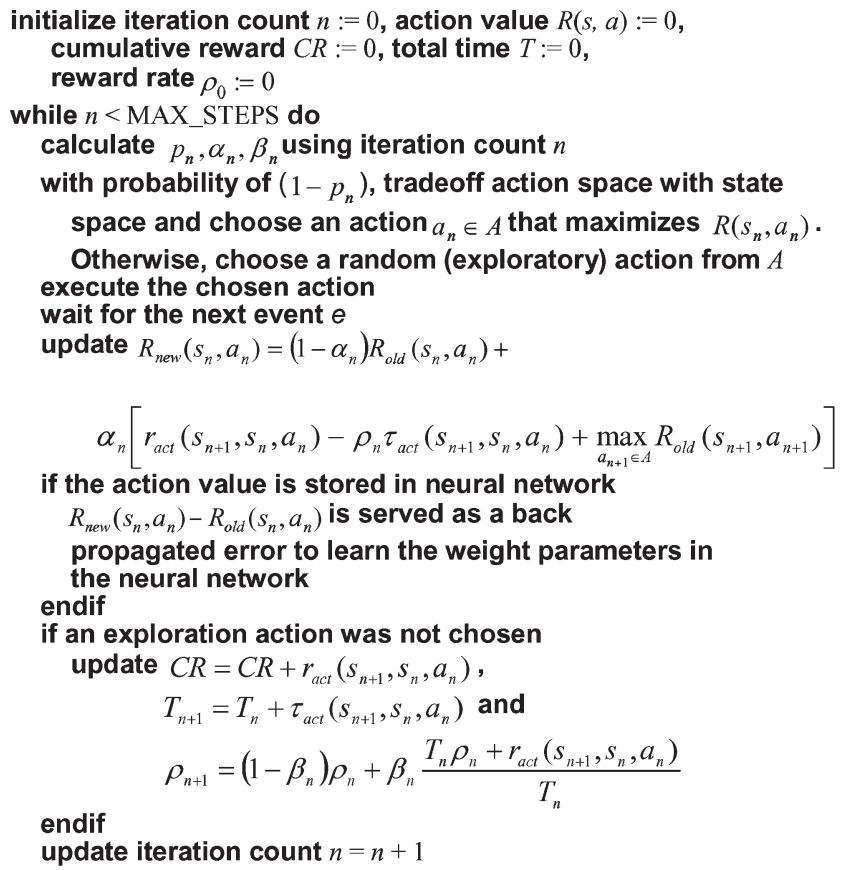

Fig. 3. Pseudo-code of the QoS provisioning scheme.

simulations. Two reward functions are used in simulations, as shown in Table I. Reward function 1 represents the scenario in which the reward generated by a call is a linear growing function with the bandwidth assigned to the call. Specifically, $r_{i j}=b_{i j}$. In reward function 2 , a convex function $r_{i j}=\left(b_{\max }^{2}-\left(b_{i j}-b_{\max }\right)^{2}\right) / b_{\max }$ is used where $b_{\max }$ is the largest bandwidth used by a call in the network. We assume that the highest possible bandwidth level is requested by the call arrival. That is, call arrival of class 1 always requests $256 \mathrm{~kb} / \mathrm{s}$ and call arrival of class 2 always requests $128 \mathrm{~kb} / \mathrm{s}$. Then, the network will make the CAC decision and decide which bandwidth level the call can use if it is admitted. Thirty percent of the offered traffic is from class 1 . Moreover, call holding time and cell residence time are assumed to follow exponential distributions with mean values of 180 and $150 \mathrm{~s}$, respectively. The probability of a user handing off to any adjacent cell is equally likely. The target maximum allowed handoff dropping probability $T P_{h d}$ is $1 \%$ for both classes. Other QoS constraints are changed in the simulations for evaluation purposes.

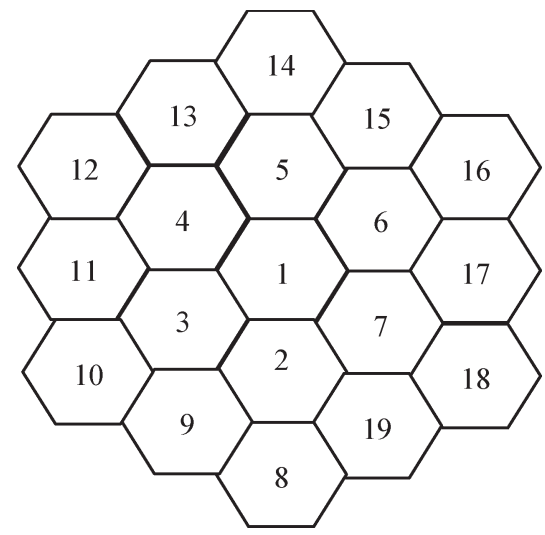

Fig. 4. Cellular network configuration used in simulations.

TABLE I

EXPERIMENTAL PARAMETERS

\begin{tabular}{|c|c|c|c|}
\hline $\begin{array}{c}\text { Traffic } \\
\text { Class }\end{array}$ & $\begin{array}{c}\text { Bandwidth } \\
\text { Level (kbps) }\end{array}$ & $\begin{array}{c}\text { Reward } \\
\text { Function 1 }\end{array}$ & $\begin{array}{c}\text { Reward } \\
\text { Function 2 }\end{array}$ \\
\hline \multirow{4}{*}{ Class 1 } & $b_{11}: 128$ & 128 & 192 \\
\cline { 2 - 4 } & $b_{12}: 192$ & 192 & 240 \\
\cline { 2 - 4 } & $b_{13}: 256$ & 256 & 256 \\
\hline Class 2 & $b_{21}: 64$ & 64 & 112 \\
\cline { 2 - 4 } & $b_{22}: 96$ & 96 & 156 \\
\hline
\end{tabular}

The constants used in the Darken-Chang-Moody decaying scheme for learning and exploration rates are chosen as $\alpha_{0}=$ $\beta_{0}=p_{0}=0.1$ and $\alpha_{r}=\beta_{r}=p_{r}=10^{11}$, which are common values in the Darken-Chang-Moody decaying scheme [37]. The monotonicity property associated with $\lambda$ is used to search for a suitable Lagrange multiplier $\lambda$. In the simulations, using binary search, we find that the handoff dropping probability constraint can be met, and the reward can be maximized when $\lambda=157560$. After considerable experimentations, the neural network used in the approximate representation is configured with 31 inputs units representing the state and action, 20 hidden units with sigmoid functions, and one output unit representing the action value. The suitable Lagrange multiplier and the numbers of input and hidden units are searched off line. Different values of the above parameters are tried in the system. After the RL approach converges after about 15 million training steps, we select the parameters that can maximize the reward and guarantee the QoS constraints. The neural network is trained on-line using the back-propagation algorithm in conjunction with the reinforcement learning. The trained network is then used to make CAC and BA decisions with different call arrival rates.

Two QoS provisioning schemes are used for comparisons: the guard channel (GC) scheme [4] for nonadaptive traffic and the ZCD02 scheme [12] for adaptive multimedia. The unit $256 \mathrm{~kb} / \mathrm{s}$ is reserved for handoff calls in the GC scheme. Zaruba et al. [12] propose an excellent approach to use simulated annealing algorithm to find the optimal call-mix selection by deriving formulas for calculating the revenue generated by calls for the service provider, given the incoming and ongoing call mixes. Both real-time and non-real-time calls are considered in ZCD02. The proposed scheme is called RL in the 


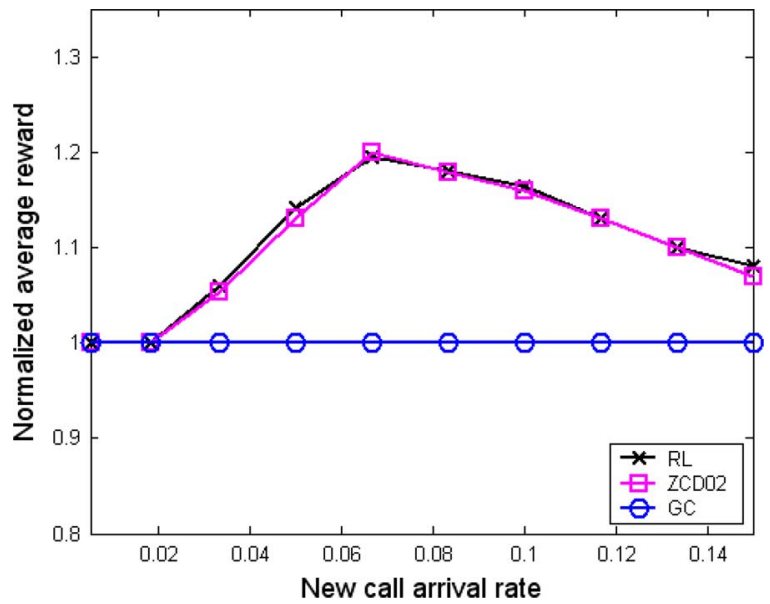

Fig. 5. Normalized average rewards.

following. The linear reward function is used in all simulation experiments, except for those in Section VI-C, where the convex reward function is used.

\section{A. Uniform Traffic}

We first use uniform traffic in simulations, where the traffic load is the same among all 19 cells. Call arrivals of both classes to each cell form a Poisson process with mean $\lambda$. The average rewards of different schemes normalized by that of the GC scheme are shown in Fig. 5. Average allocated bandwidth and intraclass fairness constraints are not considered here. We can see that RL and ZCD02 yield more rewards than GC. In the GC scheme, a call will be rejected if the free bandwidth available is not sufficient to satisfy the request. Both RL and ZCD02 have a BA function and, therefore, can yield more reward than GC. In Fig. 5, the reward of the proposed scheme is similar to that in ZCD02, because both of them can maximize network revenue in QoS provisioning. We can also observe that at low traffic load, as the new call arrival rate increases, the gain becomes more significant. This is because the heavier the offered load, the more the BA is needed when the cell is not saturated. However, when the traffic is high and the cell is becoming saturated, the performance gain of RL and ZCD02 over GC is less significant. The cost of adaptation operation is not considered, i.e., $c_{a}=0$ in Fig. 5.

Fig. 6 shows the effects of $c_{a}$, i.e., the cost of adaptation operation, when new call arrival rate is 0.067 calls/s. The reward of ZCD02 drops quickly as $c_{a}$ increases and even less than that in GC when $c_{a}=150$. In contrast, the reward drops slowly in RL. Because RL formulates $c_{a}$ in the reward function, it eliminates those actions requiring a large number of adaptation operations when $c_{a}$ is high by comparing the action values of different actions. Therefore, the proposed scheme can control the adaptation cost and, therefore, the adaptation frequency, effectively. We use $c_{a}=30$ in the following simulation experiments.

The normalized bandwidth variance VB, which is an indicator of intraclass fairness, is shown in Fig. 7. We can see that RL can keep the bandwidth variance below the target value. Because the bandwidth in GC cannot be changed, the bandwidth variance is always 0 in GC. The achievements of

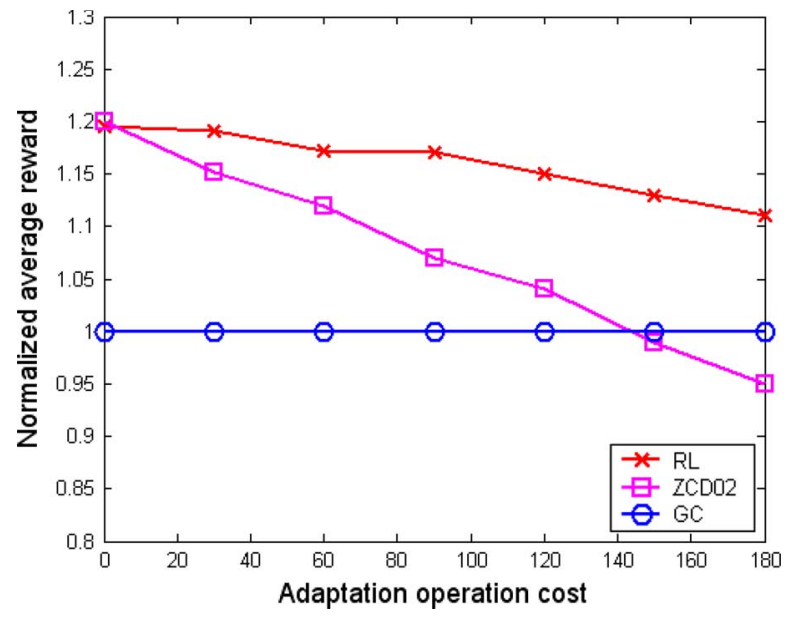

Fig. 6. Normalized average rewards versus adaptation cost.

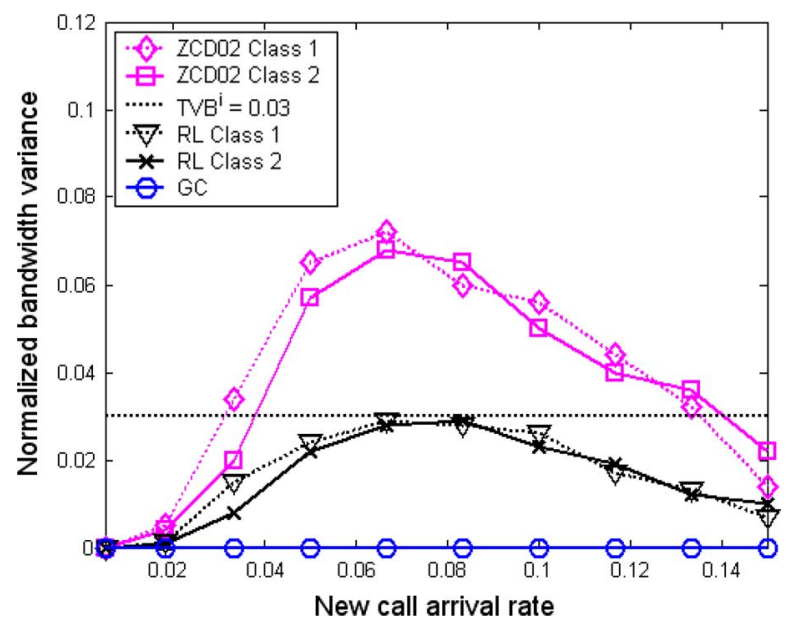

Fig. 7. Normalized bandwidth variances.

higher QoS requirements come at a cost to the system. The effects of different values of TAB and TVB on the average reward are shown in Figs. 8 and 9, respectively. We can see that a higher TAB, which is preferred from users' point of view, will reduce the reward. Similarly, a lower TVB, which means higher intraclass fairness, will reduce the reward as well. Note that although the adaptation cost and fairness are not considered in the original ZCD02, the approach in ZCD02 can be extended to consider these criteria in the call mix selection algorithm. It is expected that the adaptation cost can be limited and that the fairness constraints can be guaranteed if these criteria are considered in the extended ZCD02.

\section{B. Nonuniform Traffic}

In the nonuniform traffic situation, the cells in the second ring, i.e., cells $2-7$ in Fig. 4, have 1.5 times the new call arrival rate of those cells in the outer ring, i.e., cells $8-19$. The central cell has two times the new call arrival rate of cells in the outer ring. Since the method of predicting handoff rate from neighboring cells is not given in ZCD02, a static predicted handoff rate is used in the revenue function, and we call it ZCD02-static. Fig. 10 shows that RL yields more rewards 


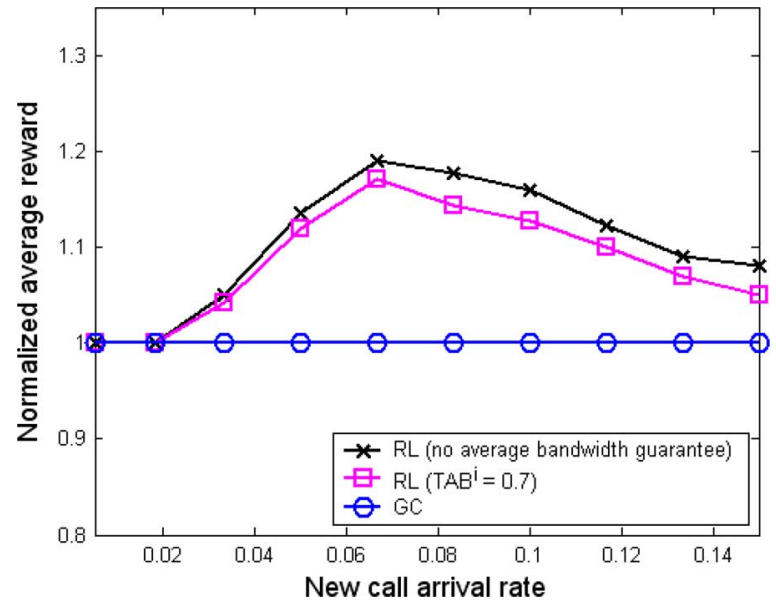

Fig. 8. Normalized average rewards for different average bandwidth requirements.

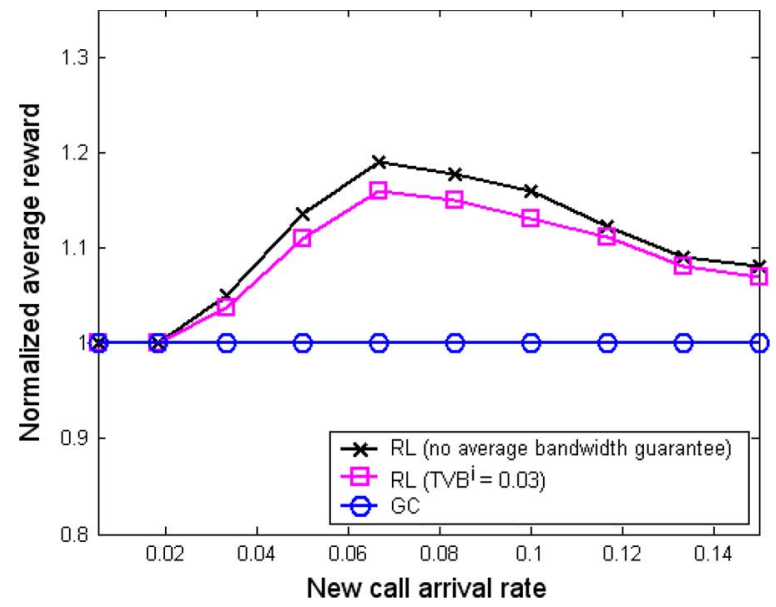

Fig. 9. Normalized average rewards for different bandwidth variance requirements.

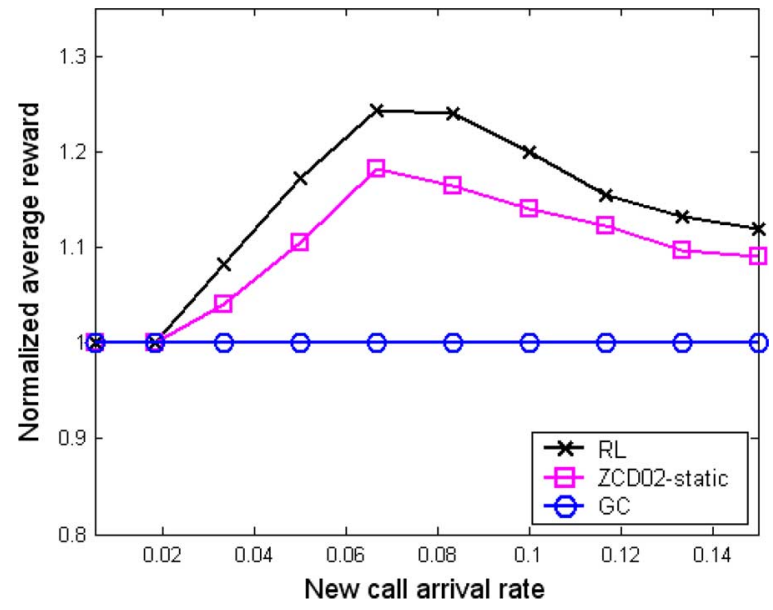

Fig. 10. Normalized average rewards with nonuniform traffic.

than the ZCD02-static and GC schemes. The performance gain of RL over GC and the difference between RL and ZCD02 static are significant in the nonuniform traffic situation. This is because our RL method takes into account the status of neighboring cells, and therefore, it can dynamically adapt to different traffic patterns.

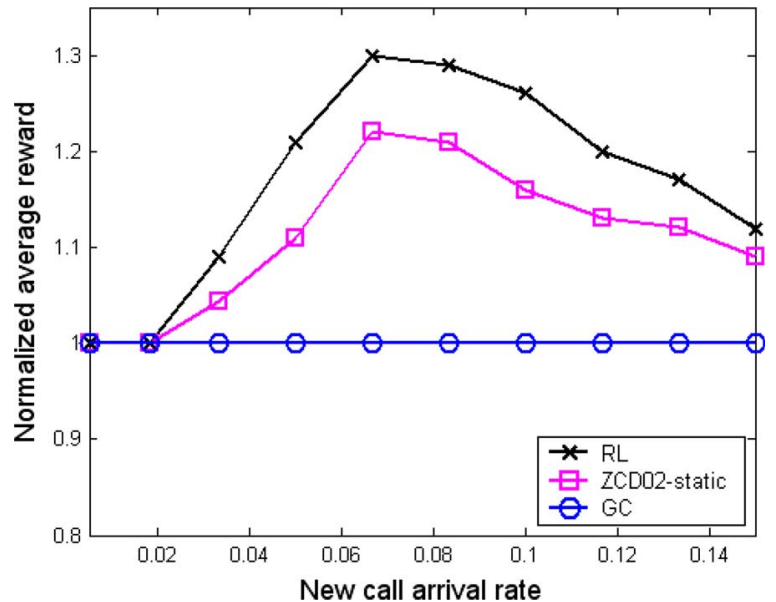

Fig. 11. Normalized average rewards with convex reward function and nonuniform traffic.

\section{Different Reward Function}

A convex reward function $r_{i j}=\left(b_{\max }^{2}-\left(b_{i j}-b_{\max }\right)^{2}\right) /$ $b_{\max }$ is used in this situation. The reward rate for specific bandwidth level of each class is shown in Table I. The simulation results using the convex reward function show a similar pattern to those using the linear reward function, and therefore, only one figure is provided here. Fig. 11 shows the average rewards of different QoS schemes with nonuniform traffic. We can see that Fig. 11 is similar to Fig. 10.

\section{Computation Complexity}

Despite the many interesting results in RL, the literature has lacked algorithms for learning optimal behavior with provably finite bounds on the computation resources (actions and computation time) [39]. The computation complexity varies for different RL algorithms. The computation resources can be polynomial in the number of states [39]. Because no proved results about the computation complexity of the used SMART RL algorithm are available, we simply calculate the average number of operations required to make one decision in both ZCD02 and the proposed scheme. Further study on the computation complexity of the proposed algorithm is in progress.

ZCD02 uses simulated annealing to find the optimal call mix, in which a variable called temperature is decreased periodically by employing a monotone descendent cooling function. We follow the example given in ZCD02, where 90 temperature steps are used, and each step is repeated 100 times. In each of the 9000 steps, the revenue and the constraints are reevaluated. In RL, because a neural network is used in the approximate representation, the major operations required to make $\mathrm{CAC}$ and BA decisions come from retrieving action values and comparing them. We run the simulations with a fixed call arrival rate of 0.1 calls/s for 1000 call arrivals and departures and calculate the average number of operations (additions, multiplications, and comparisons) required to make one decision. The number of operations is about $1.8 \times 10^{5}$ in ZCD02 and $1 \times 10^{4}$ in RL. This shows that ZCD02 will be more expensive than RL for computation resources in practice. However, training is needed 


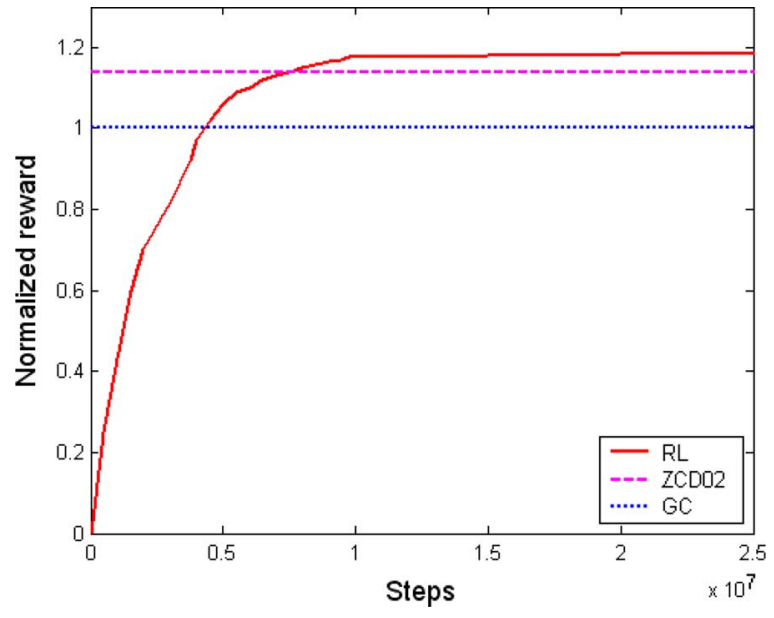

Fig. 12. Normalized average rewards versus steps.

for the RL approach. Fig. 12 shows the normalized average rewards of different schemes as time goes by. We can see the training process of the RL approach in Fig. 12. Because both the RL algorithm and the neural network need training, only after some training steps does the RL approach converge. Both ZCD02 and GC do not need any training. In this experiment, we use uniform traffic, and $c_{a}=30$.

\section{CONClusions AND Future Work}

In this paper, we have proposed a new QoS provisioning method for adaptive multimedia in cellular wireless networks. The large number of states and the difficulty to estimate the state transition probabilities in practical systems motivate us to choose a model free average reward reinforcement learning solution to solve this problem. Neural networks were used to facilitate implementation of the algorithm. By considering the status of neighboring cells, the proposed scheme can dynamically adapt to the changes in traffic condition. Three QoS constraints (handoff dropping probability, average allocated bandwidth, and intraclass fairness) have been considered. Simulation results have been presented to show the effectiveness of the proposed scheme in adaptive multimedia cellular networks.

Further study is in progress to reduce or eliminate the signaling overhead of exchanging status information by some feature extraction and local estimation functions. It is also very interesting to consider other average reward reinforcement learning algorithms [19], [38].

\section{REFERENCES}

[1] Information Technology Coding of Audio-Visual Objects: Visual, Commitee draft, ISO/IEC 144962-2, Oct. 1997.

[2] Video Coding for Low Bit Rate Communications, ITU-T H.263, 1998.

[3] 3GPP, RRC Protocol Specification, 3G TS25.331 Ver. 3.12.0, Sep. 2002.

[4] D. Hong and S. S. Rappaport, "Traffic model and performance analysis for cellular mobile radio telephone systems with prioritized and nonprioritized handoff procedures," IEEE Trans. Veh. Technol., vol. VT-35, no. 3, pp. 77-92, Aug. 1986.

[5] S. Wu, K. Y. M. Wong, and B. Li, "A dynamic call admission policy with precision QoS guarantee using stochastic control for mobile wireless networks," IEEE/ACM Trans. Netw., vol. 10, no. 2, pp. 257-271, Apr. 2002
[6] Y. B. Lin, A. Noerpel, and D. Harasty, "The sub-rating channel assignment strategy for PCS hand-offs," IEEE Trans. Veh. Technol., vol. 45, no. 1, pp. 122-130, Feb. 1996.

[7] C. Chou and K. G. Shin, "Analysis of combined adaptive bandwidth allocation and admission control in wireless networks," in Proc. IEEE INFOCOM, Jun. 2002, pp. 676-684.

[8] A. K. Talukdar, B. R. Badrinath, and A. Acharya, "Rate adaptation schemes in networks with mobile hosts," in Proc. ACM/IEEE MOBICOM, Oct. 1998, pp. 169-180.

[9] T. Kwon, J. Choi, Y. Choi, and S. Das, "Near optimal bandwidth adaptation algorithm for adaptive multimedia services in wireless/mobile networks," in Proc. IEEE VTC_Fall, Sep. 1999, pp. 874-878.

[10] Y. Xiao, P. Chen, and Y. Wang, "Optimal admission control for multiclass of wireless adaptive multimedia services," IEICE Trans. Commun., vol. E84-B, no. 4, pp. 795-804, Apr. 2001.

[11] Y. Xiao, C. L. P. Chen, and B. Wang, "Bandwidth degradation QoS provisioning for adaptive multimedia in wireless/mobile networks," Comput. Commun., vol. 25, no. 13, pp. 1153-1161, Aug. 2002.

[12] G. V. Zaruba, I. Chlamtac, and S. K. Das, "A prioritized real-time wireless call degradation framework for optimal call mix selection," Mobile Netw. Appl., vol. 7, no. 2, pp. 143-151, Apr. 2002.

[13] F. Yu, V. W. S. Wong, and V. C. M. Leung, "Efficient QoS provisioning for adaptive multimedia in mobile communication networks by reinforcement learning," in Proc. BroadNets, San Jose, CA, Oct. 2004, pp. 579-588.

[14] F. Yu, V. W. S. Wong, and V. C. M. Leung, "Efficient QoS provisioning for adaptive multimedia in mobile communication networks by reinforcement learning," Mobile Netw. Appl., vol. 11, no. 1, pp. 101-110, Feb. 2006.

[15] T. Kwon, Y. Choi, C. Bisdikian, and M. Naghshineh, "QoS provisioning in wireless/mobile multimedia networks using an adaptive framework," Wireless Netw., vol. 9, no. 1, pp. 51-59, 2003.

[16] S. Ganguly and B. Nath, "QoS provisioning for adaptive services with degradation in cellular network," in Proc. IEEE WCNC, Mar. 2003, pp. $1367-1372$.

[17] T. S. Rappaport, Wireless Communications: Principles and Practice. Englewood Cliffs, NJ: Prentice-Hall, 1996.

[18] H. Tong and T. X. Brown, "Adaptive call admission control under quality of service constraints: A reinforcement learning solution," IEEE J. Sel. Areas Commun., vol. 18, no. 2, pp. 209-221, Feb. 2000.

[19] P. Marbach, O. Mihatsch, and J. N. Tsitsiklis, "Call admission control and routing in integrated services networks using neuro-dynamic programming," IEEE J. Sel. Areas Commun., vol. 18, no. 2, pp. 197-208, Feb. 2000.

[20] S. P. Singh and D. P. Bertsekas, "Reinforcement learning for dynamic channel allocation in cellular telephone systems," in Advances in NIPS 9, M. Mozer et al., Eds. Cambridge, MA: MIT Press, 1997, pp. 974-980.

[21] J. Nie and S. Haykin, "A Q-learning based dynamic channel assignment technique for mobile communication systems," IEEE Trans. Veh. Technol., vol. 48, no. 5, pp. 1676-1687, Sep. 1999.

[22] E. Alexandri, G. Martinez, and D. Zeghlache, "A distributed reinforcement learning approach to maximize resource utilization and control handover dropping in multimedia wireless networks," in Proc. IEEE PIMRC, Sep. 2002, pp. 2249-2253.

[23] Y.-S. Chen, C.-J. Chang, and F.-C. Ren, "Q-learning-based multirate transmission control scheme for RRM in multimedia WCDMA systems," IEEE Trans. Veh. Technol., vol. 53, no. 1, pp. 38-48, Jan. 2004.

[24] D. Taubman and A. Zakhor, "A common framework for rate and distortion based scaling of highly scalable compressed video," IEEE Trans. Circuits Syst. Video Technol., vol. 6, no. 4, pp. 329-354, Aug. 1996.

[25] D. Wu, Y. T. Hou, and Y. Q. Zhang, "Scalable video coding and transport over broadband wireless networks," Proc. IEEE, vol. 89, no. 1, pp. 6-20, Jan. 2001.

[26] M. L. Puterman, Markov Decision Processes. New York: WileyInterscience, 1994.

[27] C. J. C. H. Watkins and P. Dayan, "Q-learning," Mach. Learn., vol. 8, no. 3/4, pp. 279-292, May 1992.

[28] S. Mahadevan, "Average reward reinforcement learning: Foundations, algorithms, and empirical results," Mach. Learn., vol. 22, no. 1-3, pp. 159196, Mar. 1996.

[29] T. K. Das, A. Gosavi, S. Mahadevan, and N. Marchalleck, "Solving semiMarkov decision problems using average reward reinforcement learning," Manage. Sci., vol. 45, no. 4, pp. 560-574, 1999.

[30] A. Gosavi, "An algorithm for solving semi-Markov decision problems using reinforcement learning: Convergence analysis and numerical results," Ph.D. dissertation, Univ. South Florida, Tampa, FL, 1999.

[31] A. Gosavi, N. Bandla, and T. K. Das, "A reinforcement learning approach to airline seat allocation for multiple fare classes with overbooking," IIE Trans., vol. 34, no. 9, pp. 729-742, 2002. 
[32] A. Gosavi, "Reinforcement learning for long-run average cost," Eur. J. Oper. Res., vol. 155, no. 3, pp. 654-674, Jun. 2004.

[33] F. J. Beutler and K. W. Ross, "Time-average optimal constrained semiMarkov decision processes," Adv. Appl. Prob., vol. 18, no. 2, pp. 341-359, Jun. 1986.

[34] F. J. Beutler and K. W. Ross, "Optimal policies for controlled Markov chains with a constraint," J. Math. Anal. Appl., vol. 112, no. 1, pp. 236$252,1985$.

[35] E. Altman, Constrainted Markov Decision Process. London, U.K.: Chapman \& Hall, 1999.

[36] D. P. Bertsekas and J. N. Tsitsiklis, Neuro-Dynamic Programming. Belmont, MA: Athena Scientific, 1996.

[37] C. Darken, J. Chang, and J. Moody, "Learning rate schedules for faster stochastic gradient search," in Proc. IEEE Workshop Neural Netw. Signal Process., Sep. 1992, pp. 3-12.

[38] J. Abounadi, D. Bertsekas, and V. S. Borkar, "Learning algorithms for Markov decision processes with average cost," SIAM J. Control Optim., vol. 40, no. 3, pp. 681-698, 2001.

[39] M. Kearns and S. Singh, "Near-optimal reinforcement learning in polynomial time," Mach. Learn., vol. 49, no. 2/3, pp. 209-232, Nov. 2002.

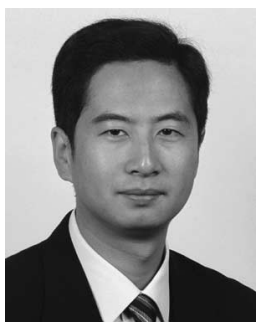

F. Richard Yu (S'00-M'03) received the Ph.D. degree in electrical engineering from the University of British Columbia (UBC), Vancouver, BC, Canada, in 2003.

From 2002 to 2004, he was with Ericsson, Lund, Sweden, where he worked on the research and development of dual-mode UMTS/GPRS handsets. From 2005 to 2006, he was with a start-up company in California, where he worked on research and development in the areas of advanced wireless communication technologies and new standards. He was a Postdoctoral Research Fellow with UBC in 2005 and 2006. In 2006, he joined the Carleton School of Information Technology and the Department of Systems and Computer Engineering at Carleton University, Ottawa, ON, Canada, where he is currently an Assistant Professor. His research interests include cross-layer design, QoS provisioning, and security in wireless networks.

Dr. Yu has served on the Technical Program Committee (TPC) of numerous conferences, i.e., the TPC Cochair of the IEEE 68th Vehicular Technology Conference (VTC-Fall 2008) Track 4 (Wireless Networks and Cognitive Radio) and the TPC Cochair of the First International Workshop on Wireless Networking for Intelligent Transportation Systems in 2007.

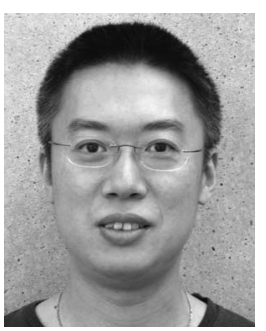

Vincent W. S. Wong (SM'07) received the B.Sc. degree from the University of Manitoba, Winnipeg, Canada, in 1994, the M.A.Sc. degree from the University of Waterloo, Waterloo, ON, Canada, in 1996, and the Ph.D. degree from the University of British Columbia (UBC), Vancouver, Canada, in 2000 .

From 2000 to 2001, he was a Systems Engineer with PMC-Sierra Inc. He is currently an Associate Professor with the Department of Electrical and Computer Engineering, UBC. His current research interests are in resource and mobility management for wireless mesh networks, wireless sensor networks, and heterogeneous wireless networks.

Dr. Wong is an Associate Editor of the IEEE TRANSACTIONS ON VEHICULAR TECHNOLOGY. He serves as TPC member of various conferences, including the IEEE International Conference on Communications (ICC) and Globecom. He received the Natural Sciences and Engineering Research Council postgraduate scholarship and the Fessenden Postgraduate Scholarship from the Communications Research Centre Industry Canada, during his graduate studies.

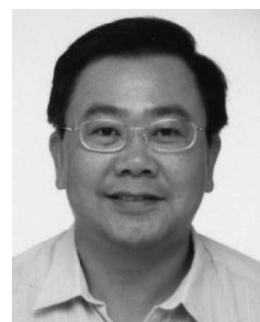

Victor C. M. Leung (S'75-M'79-SM'97-F'03) received the B.A.Sc. (Hons.) and the Ph.D. degrees in electrical engineering from the University of British Columbia (UBC), Vancouver, Canada, in 1977 and 1981 , respectively.

From 1981 to 1987, he was a Senior Member of Technical Staff at MPR Teltech Ltd., specializing in the planning, design, and analysis of satellite communication systems. In 1988, he was a Lecturer with the Department of Electronics, The Chinese University of Hong Kong, Shatin, N.T., Hong Kong. In 1989, he joined the UBC as a Faculty Member, where he is currently a Professor with the Department of Electrical and Computer Engineering, a holder of the TELUS Mobility Research Chair in Advanced Telecommunications Engineering, and a member of the Institute for Computing, Information, and Cognitive Systems. He is an Editor of the International Journal of Sensor Networks. His research interests are in the areas of architectural and protocol design and performance analysis for computer and telecommunication networks with applications in satellite, mobile, personal communications, and high-speed networks.

Dr. Leung is a Voting Member of the Association for Computing Machinery (ACM). He has served as the Technical Program Committee Vice Chair of the 2005 IEEE Wireless Communications and Networking Conference, as a General Cochair of the 2005 ACM International Symposium on Modeling, Analysis, and Simulation of Wireless and Mobile Systems, and the General Chair of QShine 2007. He is an Editor of the IEEE TRANSACTIONS ON WIRELESS COMMUNiCATIONS, an Associate Editor for the IEEE TRANSACTIONS ON COMPUTERS, and an Associate Editor for the IEEE TRANSACTIONS ON VEHICUlAR TECHNOLOGY. He is the recipient of a Canadian Natural Sciences and Engineering Research Council Postgraduate Scholarship and the 1977 Association of Professional Engineers of British Columbia Gold Medal as the head of the graduating class of the Faculty of Applied Science, UBC. 